\title{
Computed tomography of the cervical spine: comparison of image quality between a standard-dose and a low-dose protocol using filtered back-projection and iterative reconstruction
}

\author{
Fabio Becce - Yosr Ben Salah • Francis R. Verdun • \\ Bruno C. Vande Berg • Frederic E. Lecouvet • \\ Reto Meuli • Patrick Omoumi
}

Received: 6 September 2012 /Revised: 15 November 2012 / Accepted: 6 January 2013 /Published online: 29 January 2013

(C) ISS 2013

\begin{abstract}
Objective To compare image quality of a standard-dose (SD) and a low-dose (LD) cervical spine CT protocol using filtered back-projection (FBP) and iterative reconstruction (IR).

Materials and methods Forty patients investigated by cervical spine $\mathrm{CT}$ were prospectively randomised into two groups: SD (120 kVp, $275 \mathrm{mAs})$ and LD (120 kVp, $150 \mathrm{mAs}$ ), both applying automatic tube current modulation. Data were reconstructed using both FBP and sinogramaffirmed IR. Image noise, signal-to-noise (SNR) and contrast-to-noise (CNR) ratios were measured. Two radiologists independently and blindly assessed the following anatomical structures at $\mathrm{C} 3-\mathrm{C} 4$ and $\mathrm{C} 6-\mathrm{C} 7$ levels, using a four-point scale: intervertebral disc, content of neural foramina and dural sac, ligaments, soft tissues and vertebrae.
\end{abstract}

\footnotetext{
F. Becce $(\bowtie) \cdot$ R. Meuli

Department of Diagnostic and Interventional Radiology,

Centre Hospitalier Universitaire Vaudois, University

of Lausanne, Rue du Bugnon 46,

1011 Lausanne, Switzerland

e-mail: fabio.becce@chuv.ch

F. Becce $\cdot$ Y. Ben Salah • B. C. Vande Berg • F. E. Lecouvet

P. Omoumi

Department of Radiology, Cliniques Universitaires

Saint-Luc, Université Catholique Louvain,

Avenue Hippocrate 10,

1200 Brussels, Belgium

F. R. Verdun

Institute of Radiation Physics, Centre Hospitalier

Universitaire Vaudois, University of Lausanne,

Rue du Grand-Pré 1,

1007 Lausanne, Switzerland
}

They subsequently rated overall image quality using a tenpoint scale.

Results For both protocols and at each disc level, IR significantly decreased image noise and increased SNR and CNR, compared with FBP. SNR and CNR were statistically equivalent in LD-IR and SD-FBP protocols. Regardless of the dose and disc level, the qualitative scores with IR compared with FBP, and with LD-IR compared with SD-FBP, were significantly higher or not statistically different for intervertebral discs, neural foramina and ligaments, while significantly lower or not statistically different for soft tissues and vertebrae. The overall image quality scores were significantly higher with IR compared with FBP, and with LD-IR compared with SD-FBP.

Conclusion LD-IR cervical spine CT provides better image quality for intervertebral discs, neural foramina and ligaments, and worse image quality for soft tissues and vertebrae, compared with SD-FBP, while reducing radiation dose by approximately $40 \%$.

Keywords Cervical spine $\cdot$ Computed tomography $\cdot$ Filtered back-projection - Iterative reconstruction - Image quality .

Radiation exposure

\section{Introduction}

Computed tomography (CT) of the cervical spine is an alternative to magnetic resonance imaging (MRI) in the diagnosis of cervical disc herniation and/or spondylosis [1,2]. While MRI is definitely the examination of choice to assess spinal cord abnormalities, CT is more accurate for evaluating 
osteophytes and other bony changes, particularly in the case of neural foraminal stenosis $[1,2]$. Owing to both its time- and cost-effectiveness, CT is still used as a first cross-sectional imaging technique in a few countries, especially in Europe [1, 3]. It may further be performed when MRI is contraindicated and/or inconclusive. However, CT of the spine is associated with substantial radiation exposure [4].

Over the past decade, significant advances in CT technology have led to an increase in the number of indications and, consequently, radiation dose delivered to patients by $\mathrm{CT}$ examinations $[4,5]$. Therefore, CT manufacturers progressively developed several tools to manage and/or reduce radiation dose. These include automatic tube current modulation, low electronic noise detector systems and, most recently, iterative reconstruction (IR) techniques $[5,6]$. Moreover, variation in tube voltage and bismuth thyroid shields also proved to be useful in reducing radiation exposure in CT of the neck [7,8]. Besides, the advent of CT scanners with up to 320 detector rows helped to further reduce radiation dose by using the volumetric mode, which reduces over-ranging and overbeaming effects [9].

Since their implementation in clinical routine, IR methods have been extensively applied in abdominal [10], thoracic [11] and cardiovascular $[12,13] \mathrm{CT}$ imaging. These techniques allowed the radiation dose to be substantially reduced, while maintaining nearly constant diagnostic image quality. One feature of the musculoskeletal system is its varied composition of both soft (ligament, muscle, tendon, fat) and dense (bone) tissues. To our knowledge, the impact of IR on the conspicuity of these different anatomical structures, each evaluated separately, has not been assessed so far.

Thus, the aim of our study was to quantitatively and qualitatively compare the overall image quality and the conspicuity of different anatomical structures between a standard-dose and a low-dose cervical spine CT protocol using both filtered back-projection (FBP) and IR. We mainly focused on the comparison between the standard-dose FBP and low-dose IR protocol.

\section{Materials and methods}

This single-centre prospective study was approved by the institutional ethics committee and all patients gave written informed consent.

Patients

From October to November 2011, 45 consecutive patients with chronic neck pain and/or cervical radiculopathy were investigated by unenhanced $\mathrm{CT}$ of the cervical spine in our radiology department. Three patients were excluded owing to substantial metallic artefacts caused by surgical material, while two refused to take part in the study. Thus, the final study population consisted of 40 patients ( 28 women, 12 men; mean age 53.1 years, range 18 80 ), who were randomly assigned to one of the following two groups: standard-dose CT $(n=20 ; 14$ women, 6 men; mean age 51.6 years, range $25-80$ ), or low-dose CT $(n=20 ; 14$ women, 6 men; mean age 54.5 years, range 18-77). As an indicator of the patient's morphotype, the anteroposterior (AP) diameter of the neck was measured parallel to the intervertebral disc at the C6-C7 level on the lateral CT scout view, and measurements of the two groups were compared.

\section{CT protocol}

All unenhanced CT examinations were performed on a 40detector row CT scanner (Somatom Definition AS; Siemens Healthcare, Forchheim, Germany). Patients were positioned supine, with the head first on the CT table. Data acquisition was obtained from the $\mathrm{C} 3$ to $\mathrm{T} 1$ vertebrae, using the following parameters: tube voltage, $120 \mathrm{kVp}$; reference tube current-time product, 275 or $150 \mathrm{mAs}$ in the standard-dose or low-dose protocol respectively; effective tube current-time product, 178-305 or 120-201 mAs, respectively (by applying Care Dose 4D; Siemens Healthcare); detector configuration, $40 \times 0.6 \mathrm{~mm}$; pitch, 0.8 ; gantry rotation time, $1 \mathrm{~s}$. The low-dose settings were inspired by the lowest-dose protocols reported in the literature for cervical spine CT $[14,15]$, as well as by our prior experience on the subject (personal unpublished data).

The CT raw data were reconstructed by using both a conventional FBP and an IR (sinogram-affirmed iterative reconstruction, SAFIRE; Siemens Healthcare) algorithm in the standard-dose (Fig. 1) and the low-dose (Fig. 2) protocol. SAFIRE is a recently introduced second-generation IR technique, whose process of image reconstruction has been presented in detail elsewhere $[12,13]$. As recommended by the manufacturer and used in other clinical studies [12, 13], we applied a medium strength level of IR (i.e. SAFIRE 3 ) in this study. The following image reconstruction parameters were used: field-of-view (FOV), $12 \times 12 \mathrm{~cm}$; section thickness/increment, $0.75 / 0.75 \mathrm{~mm}$; soft tissue (B41s for FBP and I41s for IR algorithms respectively) and bone (B70h and $\mathrm{I} 70 \mathrm{~h}$ respectively) convolution kernels. The overall image reconstruction time was approximately 20 and $60 \mathrm{~s}$ for the FBP and SAFIRE 3 examinations respectively.

\section{Noise power spectrum analysis}

In order to characterise the spatial frequency bandwidth transferred with FBP (B41s convolution kernel) and SAFIRE 3 (I41s convolution kernel), the noise power spectrum (NPS) of CT images of a $20-\mathrm{cm}$ diameter homogeneous phantom filled 
Fig. 1 A 50-year-old woman with chronic neck pain, who belongs to the standard-dose group. Axial (C3-C4 level) and sagittal-reformatted unenhanced $\mathrm{CT}$ images (window level/width, 60/300) of the cervical spine, reconstructed with $\mathbf{a}, \mathbf{b}, \mathbf{e}, \mathbf{f}$ filtered back-projection (FBP) and $\mathbf{c}, \mathbf{d}, \mathbf{g}, \mathbf{h}$ sinogramaffirmed iterative reconstruction (SAFIRE, strength level 3), using soft tissue (a-d) and bone convolution kernels (e-h). a Three region-of-interests (ROI) of $50 \mathrm{~mm}^{2}$ each are drawn in the intervertebral disc (1), the spinal cord (2) and posterior paraspinal muscles (3). Note the change in conspicuity of the different anatomical structures with iterative reconstruction (IR), compared with FBP
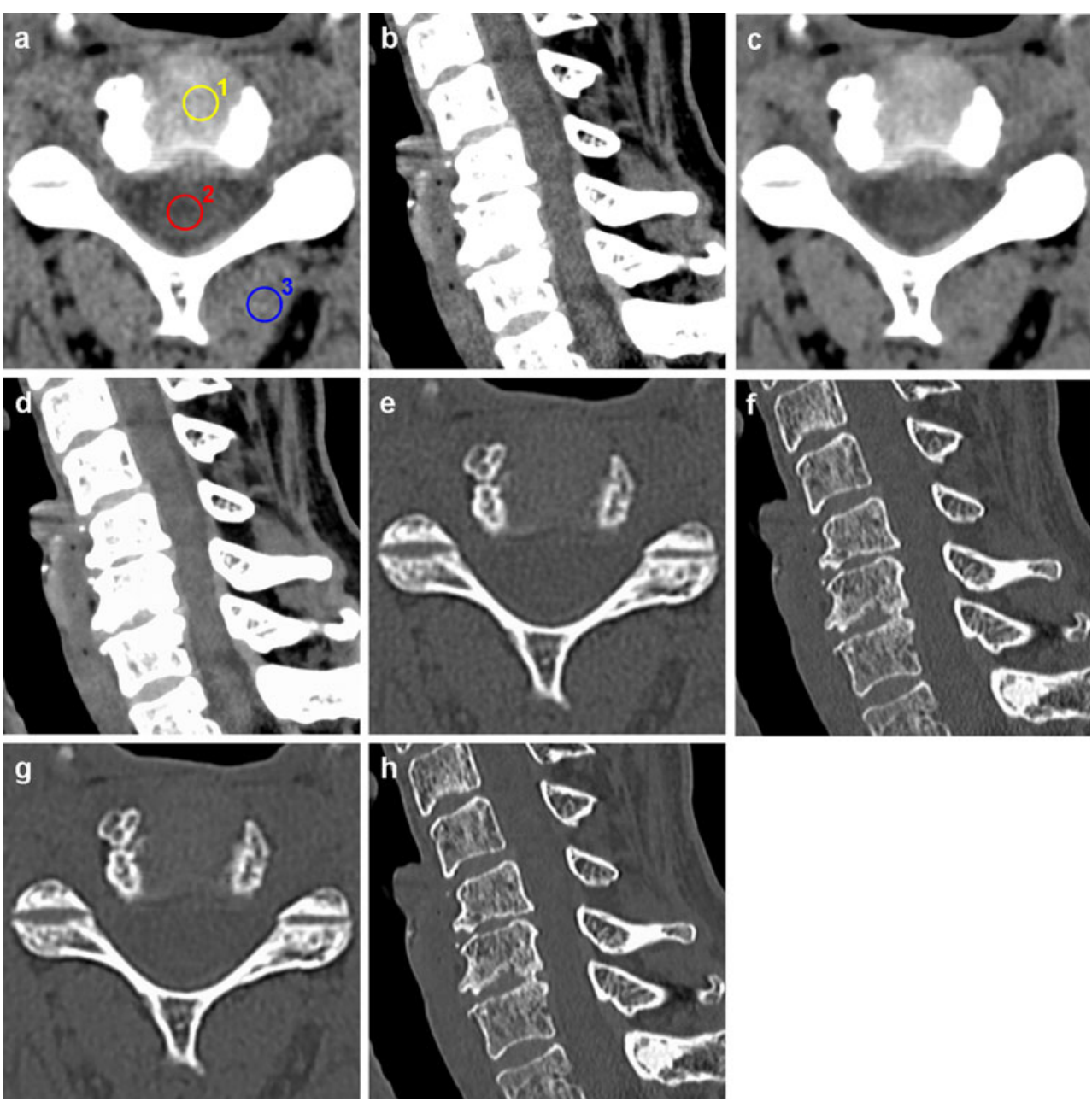

with water were calculated. For both reconstruction algorithms, 100 images were acquired using a similar FOV as for clinical CT examinations with a volume CT dose index $\left(\mathrm{CTDI}_{\mathrm{vol}}\right)$ set to $10 \mathrm{mGy}$. The NPS analysis was then performed using four ROIs of $64 \times 64$ pixels extracted from each reconstructed image. Each ROI overlapped its direct neighbours by 16 pixels in both horizontal and vertical directions.

\section{Radiation exposure estimations}

The $\mathrm{CTDI}_{\mathrm{vol}}$ (expressed in the 16-cm diameter CTDI phantom) and the dose-length product (DLP) were automatically generated by the CT unit and archived. The effective dose (ED) was estimated by multiplying the DLP by the appropriate conversion coefficient $\left(0.0051 \mathrm{mSv} \times \mathrm{mGy}^{-1} \times \mathrm{cm}^{-1}\right.$ for an adult, neck region and $120 \mathrm{kV}$ [16]).

\section{Image analysis}

Two musculoskeletal radiologists (observers 1 and 2, with 7 and 4 years of experience in spine imaging respectively, working in two different institutions) independently reviewed all CT examinations on a picture archiving and communication system (PACS) workstation (Carestream Client version 11.3; Carestream Health, Rochester, NY, USA). They were both blinded to CT parameters and image reconstruction algorithms, and examinations were displayed in a random order.

\section{Quantitative analysis}

All measurements were performed by observer 2. Three region-of-interests (ROI) of $50 \mathrm{~mm}^{2}$ each were placed in the most homogeneous area of the intervertebral disc, the spinal cord, and the posterior paraspinal muscles (i.e. multifidus or semispinalis capitis) on axial $\mathrm{CT}$ images at both C3-C4 and C6-C7 levels (Fig. 1a). In order to limit partial volume effects, care was taken to avoid other structures present on adjacent sections. Furthermore, the ROI were directly copied and pasted from FBP to SAFIRE 3 images to be exactly in the same position. Measurements were repeated on two consecutive sections and CT numbers $\left(\mathrm{CT}_{\mathrm{n}}\right.$, i.e. Hounsfield unit, $\left.\mathrm{HU}\right)$ averaged. Image noise, defined as the standard deviation (SD) of the mean $\mathrm{CT}_{n}$ 
Fig. 2 A 77-year-old man with right $\mathrm{C} 4$ radiculopathy, who belongs to the low-dose group. Axial (C3-C4 level) and sagittal-reformatted unenhanced $\mathrm{CT}$ images (window level/width, 60/300) of the cervical spine, reconstructed with $\mathbf{a}, \mathbf{b}, \mathbf{e}, \mathbf{f}$ filtered back-projection (FBP) and $\mathbf{c}, \mathbf{d}, \mathbf{g}, \mathbf{h}$ sinogramaffirmed iterative reconstruction (SAFIRE, strength level 3), using $\mathbf{a}-\mathbf{d}$ soft tissue and $\mathbf{e}-$ h bone convolution kernels. Note the change in conspicuity of the different anatomical structures with IR, compared with FBP
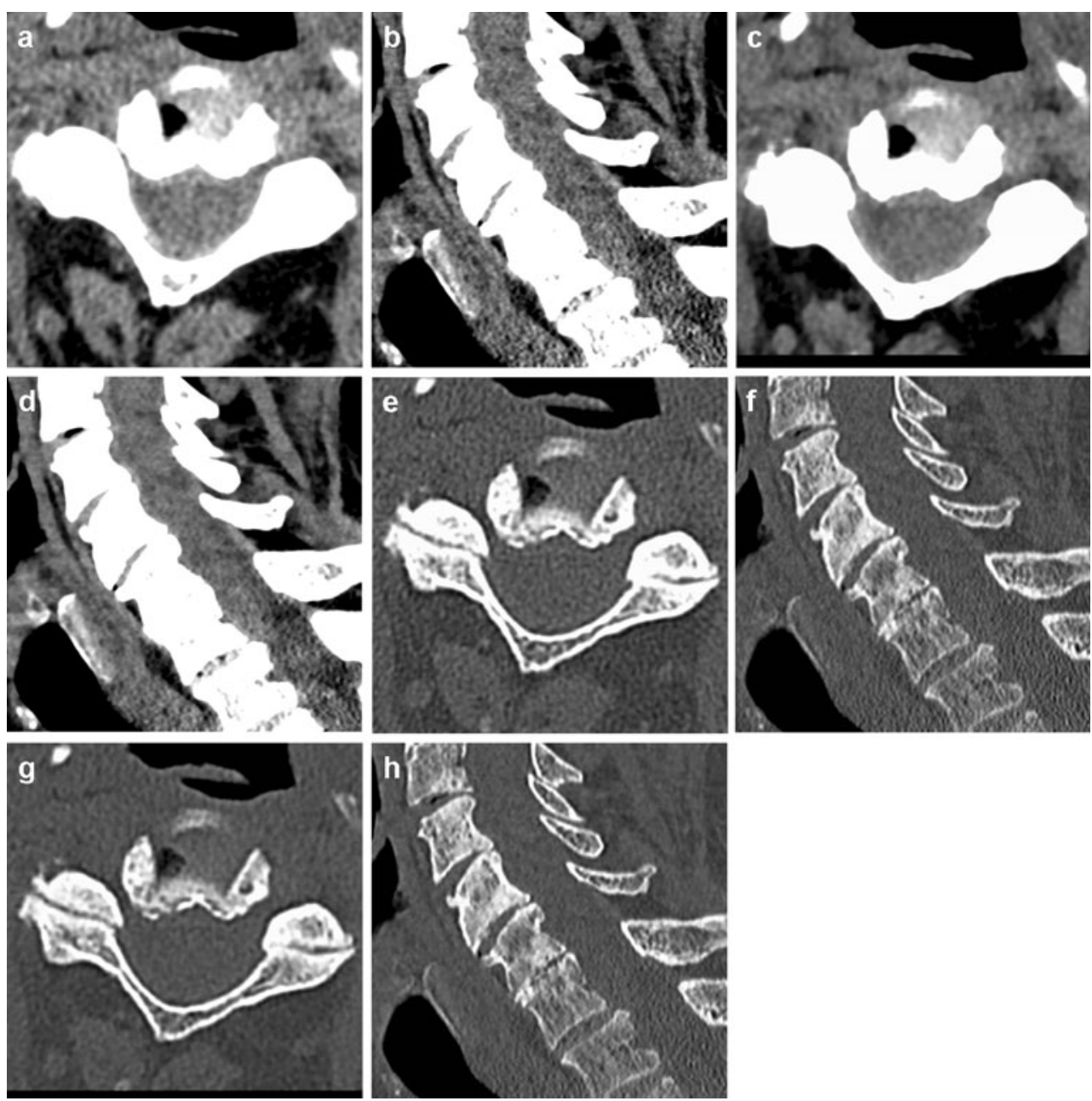

within a ROI, was measured in the posterior paraspinal muscles. The signal-to-noise (SNR) and contrast-to-noise $(\mathrm{CNR})$ ratios were calculated as follows:

- $\mathrm{SNR}=$ mean $\mathrm{CT}_{\mathrm{n}} / \mathrm{SD}$, within the ROI placed in the posterior paraspinal muscles

- $\mathrm{CNR}=\Delta$ mean $\mathrm{CT}_{\mathrm{n}} /[(\Sigma \mathrm{SD}) / 2]$, within the ROI placed in the intervertebral disc and the spinal cord

\section{Qualitative (semi-quantitative) analysis}

Before starting the analysis, both observers were instructed on the image grading system with five test cases that were not included in the study. All CT images were displayed with the window level/width set to $60 / 300$ for the soft tissues and 400/2,000 for bone respectively. The conspicuity of the following anatomical structures was assessed using a four-point scale $(4=$ excellent; 3 =good; 2 = moderate; $1=$ poor), at both $\mathrm{C} 3-\mathrm{C} 4$ and $\mathrm{C} 6-\mathrm{C} 7$ levels: the intervertebral disc, the content of neural foramina (i.e. the spinal nerve roots and ganglia) and of the dural sac (i.e. the spinal cord), the flavum and posterior longitudinal ligaments, the subcutaneous tissue and muscles (including intermuscular spaces), and the trabecular bone of vertebrae. In addition, the presence of photon starvation (streak) artefacts was evaluated using a four-point scale $(4=$ no artefact; $3=$ minor artefacts; $2=$ moderate artefacts, not reducing diagnostic acceptability; 1 = major artefacts, unacceptable). Finally, the overall image quality (in terms of diagnostic acceptability) was rated using a ten-point scale $(10=$ excellent to $1=$ poor). This image grading system was inspired by the European guidelines on quality criteria for computed tomography [17].

\section{Statistical analysis}

All data were processed using a statistical software package (MedCalc version 11.6; MedCalc Software, Mariakerke, Belgium). Patients' characteristics in both groups were compared using the Chi-squared and Student $t$ tests for unpaired samples. Continuous variables (quantitative analysis) were compared using the Student $t$ test for unpaired samples (all variables were distributed normally according to the 
Kolmogorov-Smirnov test). Ordinal variables (qualitative analysis) were compared using the Mann-Whitney $U$ test and its extension, the Kruskal-Wallis test. Given that a nonstatistically significant difference in a Student $t$ test is not a proof of equivalence [18], an equivalence test was further performed to compare the standard-dose FBP and low-dose IR protocols. Interobserver agreement was assessed by calculating weighted kappa coefficients (with linear weighting), and interpreted as follows: $\leq 0=$ poor, $0.01-0.20=$ slight, $0.21-0.40=$ fair, $0.41-0.60=$ moderate, $0.61-0.80=$ substantial, and $\geq 0.81=$ almost perfect agreement. A significance level of $p \leq 0.05$ was considered for all tests.

\section{Results}

\section{Patient groups}

There was no significant difference in gender $(p=0.73)$, age $(p=0.54)$ and AP diameter of the neck $(\Delta=0.9 \mathrm{~cm}, p=0.07)$ between the standard-dose and low-dose patient groups.

\section{Noise power spectrum}

As shown in Fig. 3, the centroids of the spatial frequency range obtained with FBP and SAFIRE 3 are very close to one another. However, the maximum value of NPS is reached at a slightly lower frequency with SAFIRE 3.

\section{Radiation exposure estimations}

The mean CTDI $_{\mathrm{vol}}$, DLP and ED were $39.0 \mathrm{mGy}$, $473.7 \mathrm{mGy} \times \mathrm{cm}$ and $2.42 \mathrm{mSv}$ for the standard-dose protocol, compared with $22.9 \mathrm{mGy}, 275.3 \mathrm{mGy} \times \mathrm{cm}$ and $1.40 \mathrm{mSv}$ for the low-dose protocol respectively (Table 1). The differences in $\mathrm{CTDI}_{\mathrm{vol}}$, DLP and ED were all statistically significant (all $p<0.01$ ).

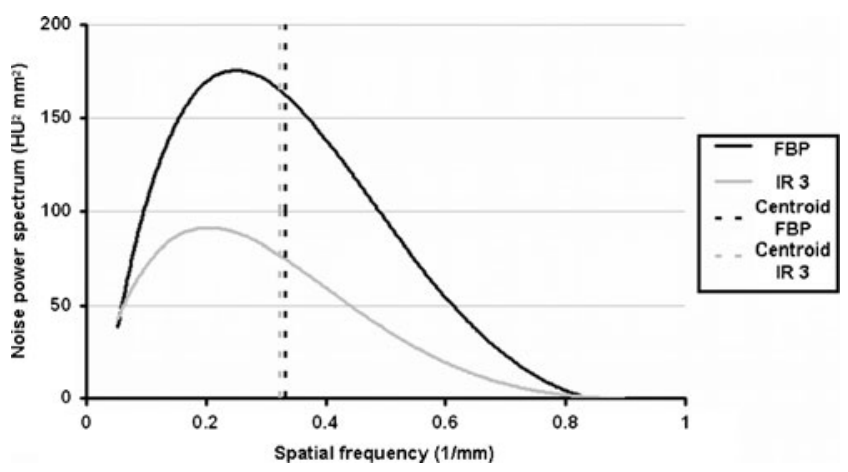

Fig. 3 Line graph illustrates the noise power spectrum (NPS) with filtered back-projection (FBP) and sinogram-affirmed iterative reconstruction (SAFIRE, strength level 3). SAFIRE 3 drastically reduces image noise while avoiding oversmoothing of the data, compared with FBP.
Quantitative analysis

The results of the quantitative analysis are reported in Table 1 and Fig. 4.

For both standard-dose and low-dose protocols, and at the $\mathrm{C} 3-\mathrm{C} 4$ and $\mathrm{C} 6-\mathrm{C} 7$ levels, the application of IR significantly decreased image noise and increased SNR and CNR, compared with FBP (all $p \leq 0.02$ ). Mean noise levels were significantly higher at $\mathrm{C} 6-\mathrm{C} 7$ than $\mathrm{C} 3-\mathrm{C} 4$ for both acquisition protocols and each image reconstruction algorithm (all $p \leq 0.04$ ).

The low-dose IR and standard-dose FBP protocols were statistically equivalent at each disc level in terms of SNR and CNR (all $p \leq 0.01$, with a tolerance interval of \pm 2 ). These two protocols were also equivalent in terms of image noise at the $\mathrm{C} 3-\mathrm{C} 4$ level $(p=0.02$, with a tolerance interval of \pm 2$)$, but neither a statistical equivalence nor a difference was found at $\mathrm{C} 6-\mathrm{C} 7$ ( $p=0.11$ and 0.60 for difference and equivalence tests, respectively, with a tolerance interval of $+/-2$ ).

\section{Qualitative (semi-quantitative) analysis}

The results of the qualitative analysis are illustrated in Fig. 5. They were comparable for both observers and for each disc level, except as described below.

For both standard-dose and low-dose protocols, the scores obtained with IR for the intervertebral discs, content of the neural foramina, and ligaments were significantly higher or not statistically different compared with FBP. As regards intervertebral discs, the scores with IR were significantly higher than with FBP (all $p \leq 0.01$ ), except for observer 2 at C6-C7 level with the low-dose protocol ( $p=$ 0.15 ). As regards the content of neural foramina, no significant difference was found between IR and FBP (all $p \geq$ 0.22 ), except for observer 1 at both disc levels with the standard-dose protocol (IR significantly better than FBP, all $p \leq 0.02$ ), and at the $\mathrm{C} 3-\mathrm{C} 4$ level with the low-dose protocol (IR significantly better, $p=0.03$ ). As regards ligaments, there was no significant difference between IR and FBP (all $p \geq 0.06$ ), except at the C3-C4 level with the lowdose protocol (IR significantly better, $p \geq 0.02$ ). For these three anatomical structures, the low-dose IR protocol received significantly higher scores than the standard-dose FBP (all $p \leq 0.05$ ).

Besides, the scores of the standard-dose and low-dose protocols obtained with IR and FBP for the content of the dural sac were comparable (all $p \geq 0.06$ ).

In contrast, the scores obtained with IR for the soft tissues and vertebrae were significantly lower or not statistically different compared with FBP, for both standard-dose and low-dose protocols. As regards soft tissues, the scores with IR were significantly lower than with FBP (all $p \leq 0.04$ ). As regards vertebrae, the scores with IR were also significantly 
Table 1 Objective image quality and radiation exposure for the standard-dose and low-dose CT protocols using filtered backprojection (FBP) and iterative reconstruction (IR)

SNR signal-to-noise ratio; $C N R$ contrast-to-noise ratio; $C T D I_{\text {vol }}$ volume $\mathrm{CT}$ dose index; $D L P$ dose-length product; $E D$ effective dose; $H U$ Hounsfield unit; CI confidence interval

\begin{tabular}{|c|c|c|c|c|c|c|}
\hline Parameter & & Level & Low-dose FBP & Low-dose IR & Standard-dose FBP & Standard-dose IR \\
\hline \multirow[t]{6}{*}{ Image quality } & \multirow{2}{*}{$\begin{array}{l}\text { Noise, } \mathrm{HU}, \\
\text { mean }(95 \% \mathrm{CI})\end{array}$} & $\mathrm{C} 3-\mathrm{C} 4$ & $13.8(12.3-15.2)$ & $9.6(8.5-10.8)$ & $9.7(8.8-10.5)$ & $7.3(6.4-8.1)$ \\
\hline & & $\mathrm{C} 6-\mathrm{C} 7$ & $21.3(17.2-25.4)$ & $15.0(12.2-17.7)$ & $12.4(10.7-14.1)$ & $8.8(7.6-10.0)$ \\
\hline & \multirow{2}{*}{$\begin{array}{l}\text { SNR, mean } \\
\quad(95 \% \mathrm{CI})\end{array}$} & $\mathrm{C} 3-\mathrm{C} 4$ & $4.1(3.6-4.6)$ & $6.0(5.2-6.8)$ & $6.0(5.1-6.9)$ & $7.8(6.9-8.6)$ \\
\hline & & $\mathrm{C} 6-\mathrm{C} 7$ & $2.9(2.3-3.5)$ & $4.1(3.3-4.9)$ & $4.8(4.0-5.6)$ & $6.7(5.6-7.9)$ \\
\hline & \multirow{2}{*}{$\begin{array}{r}\text { CNR, mean } \\
(95 \% \mathrm{CI})\end{array}$} & $\mathrm{C} 3-\mathrm{C} 4$ & $4.6(3.8-5.3)$ & $6.7(5.7-7.7)$ & $6.2(5.4-7.0)$ & $8.6(7.4-9.7)$ \\
\hline & & $\mathrm{C} 6-\mathrm{C} 7$ & $3.9(3.2-4.4)$ & $5.7(4.8-6.6)$ & $6.0(5.3-6.7)$ & $8.1(7.4-8.8)$ \\
\hline \multirow[t]{3}{*}{ Radiation exposure } & $\begin{array}{l}\mathrm{CTDI}_{\mathrm{vol}}, \mathrm{mGy} \\
\text { mean }(95 \% \mathrm{CI})\end{array}$ & & \multicolumn{2}{|l|}{$22.9(21.7-24.1)$} & \multicolumn{2}{|l|}{$39.0(37.2-40.8)$} \\
\hline & $\begin{array}{l}\text { DLP, mGy } \times \mathrm{cm} \text {, } \\
\text { mean }(95 \% \mathrm{CI})\end{array}$ & & \multicolumn{2}{|c|}{$275.3(246.7-303.9)$} & \multicolumn{2}{|l|}{$473.7(414.4-533.0)$} \\
\hline & $\begin{array}{l}\text { ED, mSv, mean } \\
(95 \% \mathrm{CI})\end{array}$ & & \multicolumn{2}{|l|}{$1.40(1.26-1.54)$} & \multicolumn{2}{|l|}{$2.42(2.12-2.72)$} \\
\hline
\end{tabular}

lower than with FBP (all $p \leq 0.01$ ), except for observer 2 at both disc levels with the low-dose protocol (all $p \geq 0.26$ ), and for observer 1 at the $\mathrm{C} 6-\mathrm{C} 7$ level with the standard-dose protocol $(p=0.15)$. For these two anatomical structures, the low-dose IR protocol received significantly lower scores than the standard-dose FBP (all $p \leq 0.02$ ), except for observer 1 at C6-C7 level $(p>0.99)$.

Furthermore, the scores of the standard-dose and lowdose protocols obtained with IR and FBP for photon starvation artefacts were comparable (all $p \geq 0.53$ ). Regardless of the protocol, no significant difference in the frequency of those artefacts was found, either at the $\mathrm{C} 3-\mathrm{C} 4$ or the $\mathrm{C} 6-\mathrm{C} 7$ level (all $p \geq 0.12$ ).

Finally, the overall image quality scores obtained with IR were significantly higher than with FBP, for both standarddose and low-dose protocols (all $p<0.01$ ). The low-dose IR protocol received significantly higher scores than the standard-dose FBP (all $p<0.01$ ).

Interobserver agreement was substantial for the low-dose IR $(\kappa=0.73)$ and both standard-dose protocols $(\kappa=0.66$ and
Fig. 4 Bar charts illustrate the impact of filtered backprojection $(F B P)$ and iterative reconstruction $(I R$, strength level 3) on image noise (in Hounsfield units, $H U$ ), signalto-noise $(S N R)$ and contrast-tonoise $(C N R)$ ratios, for standard-dose and low-dose CT protocols, at a $\mathrm{C} 3-\mathrm{C} 4$ and $\mathbf{b}$ C6-C7 levels. Error bars represent $95 \%$ confidence intervals

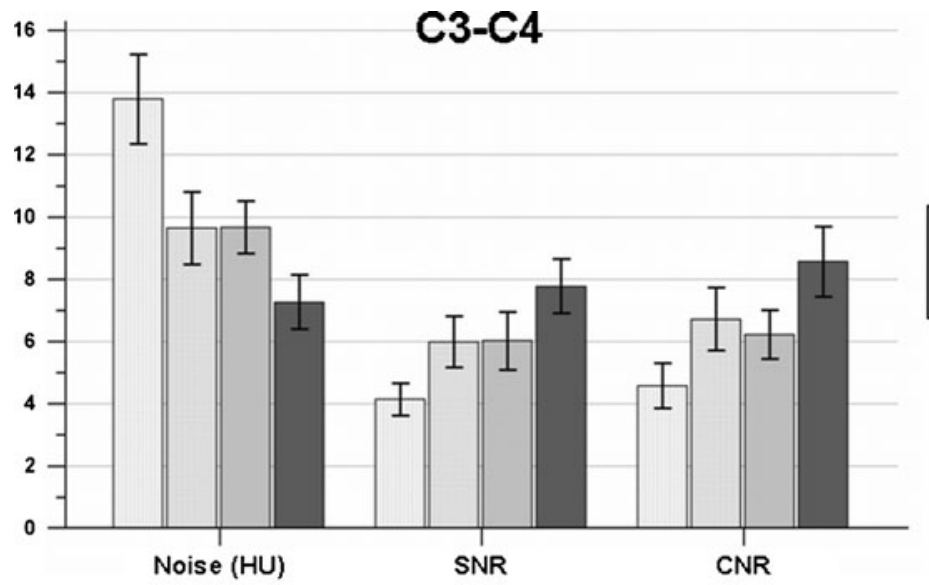

a

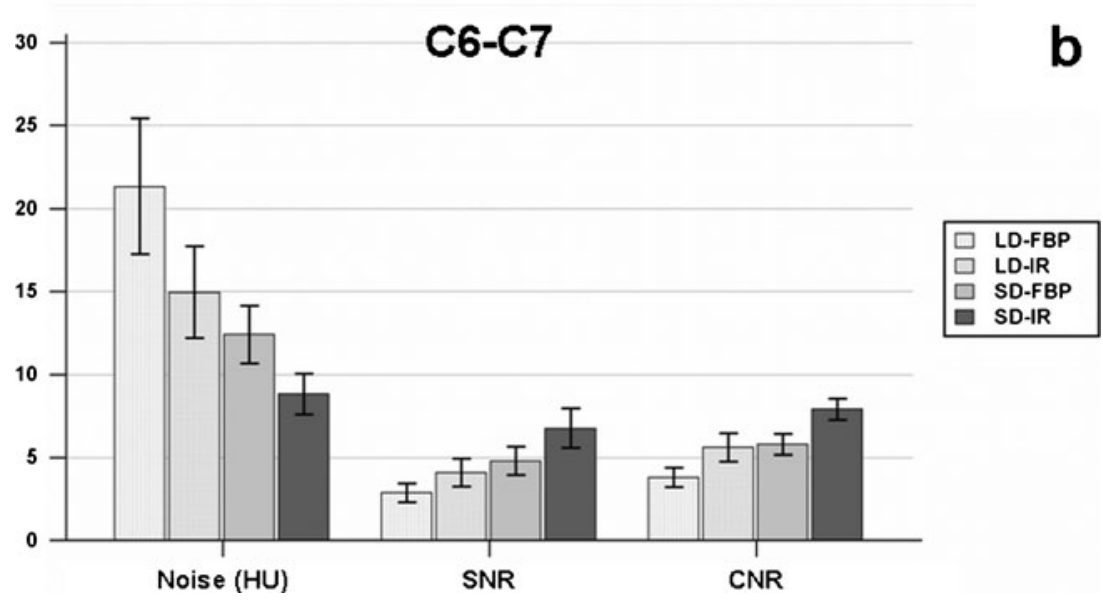




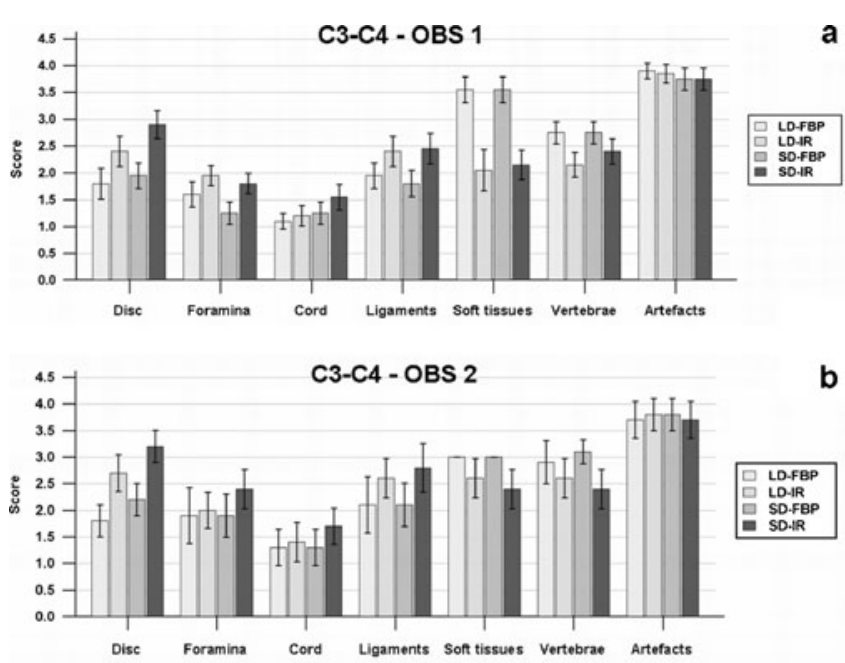

a

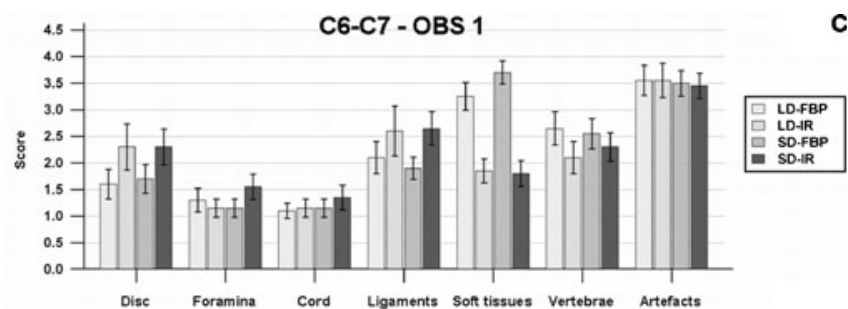

b

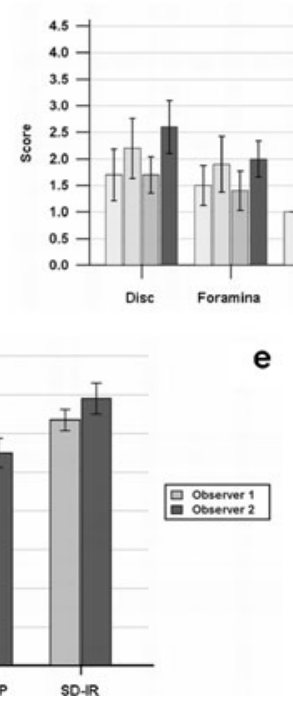

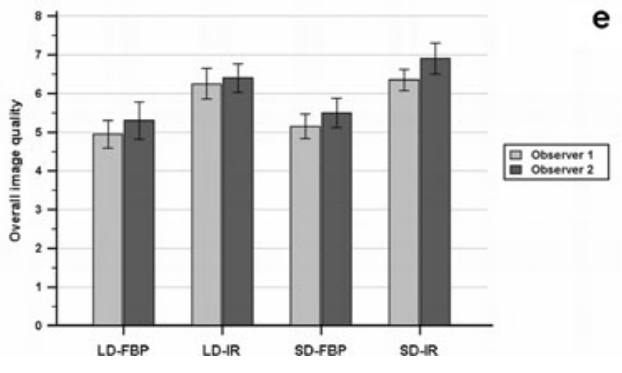

Fig. 5 Bar charts illustrate the impact of FBP and IR (strength level 3) on image quality scores for a-d different anatomical structures and photon starvation artefacts, and e overall diagnostic image quality. a

0.79 with IR and FBP respectively), while it was almost perfect for the low-dose FBP protocol $(\kappa=0.81)$.

\section{Discussion}

This patient-based study demonstrates that the application of IR in cervical spine CT enhances image quality both quantitatively and qualitatively. However, its impact depends on the anatomical structure to be analysed. Both observers found that the overall diagnostic image quality was significantly better with IR, compared with FBP (Fig. 5e). When focusing on different anatomical structures, they noted that the conspicuity of the intervertebral discs, the content of the neural foramina, and the ligaments was significantly higher (or not statistically different) with IR (Fig. 5a-d). This finding might be explained by the higher CNR obtained with IR (Table 1, Fig. 4), and is important because all these structures need to be accurately assessed in patients with chronic neck pain and/or cervical radiculopathy [1-3]. In contrast, both soft tissues and vertebrae received significantly lower (or not statistically different) scores with IR (Fig. 5a-d). Therefore, conventional FBP images should still be evaluated when assessing soft tissues and the trabecular bone of the vertebrae.
C3-C4 level, observer (OBS) 1; b C3-C4 level, OBS 2; c C6-C7 level, OBS 1; d C6-C7 level, OBS 2. Error bars represent $95 \%$ confidence intervals

Radiation exposure related to imaging studies, particularly CT, is a growing concern [19]. Among the tools implemented to reduce radiation dose in CT imaging, IR techniques have recently proved to be effective $[5,6$, 10-13]. Compared with traditional FBP algorithms, their main advantage lies in the lower noise level of reconstructed images, without sacrificing spatial resolution. Consequently, both SNR and CNR are improved. Iterative reconstruction methods can be used either to enhance image quality or to reduce radiation dose. Given that current CT technology provides diagnostic image quality for all anatomical regions, most recent studies focused on the latter objective [10-13]. Indeed, significant dose reductions of up to $66 \%$ were achieved in thoracic and abdominal $\mathrm{CT}$, while preserving acceptable diagnostic image quality [10-13]. To date, only one study has assessed the impact of IR in CT of the musculoskeletal system [20]. Focusing on the lumbar spine, Gervaise et al. demonstrated that a dose reduction of approximately $52 \%$ was achievable by applying adaptive iterative dose reduction (AIDR; Toshiba, Tochigi, Japan), while preserving diagnostic image quality [20]. Similarly, we found that the application of SAFIRE (Siemens Healthcare) in CT of the cervical spine allowed for an average $42 \%$ reduction in 
radiation dose (Table 1), while maintaining diagnostic image quality. Indeed, when comparing standard-dose FBP and low-dose IR protocols, we noted that objective image quality was kept almost constant, as the mean noise, SNR and CNR of the two protocols were comparable (Table 1, Fig. 4). Interestingly, both observers found that overall diagnostic image quality was significantly better with low-dose IR than with standard-dose FBP (Fig. 5e). Further studies are necessary to determine the highest level of dose reduction, which may depend on the anatomical region to be examined.

In order to standardise the local practices, diagnostic reference levels (DRL) have been established for most CT examinations [21]. In this context, we aimed to compare the standard-dose protocol provided by the manufacturer for cervical spine CT $(120 \mathrm{kVp}, 275 \mathrm{mAs})$ with the lowestdose protocol reported in the literature $(120 \mathrm{kVp}, 150 \mathrm{mAs})$ $[14,15]$. A second-generation IR technique (SAFIRE; Siemens Healthcare) was applied to the latter protocol to enhance its image quality. While we opted rather to reduce tube current-time product, Hoang et al. recently reported that lowering tube voltage (from 120 to $80 \mathrm{kVp}$ ) in CT of the neck resulted in a greater than $50 \%$ radiation dose reduction, without impairing subjective image quality [7]. Further studies should focus on optimising both $\mathrm{kVp}$ and $\mathrm{mAs}$ values. Lowering $\mathrm{kVp}$ would indeed allow the radiation dose to be further reduced, with a concomitant increase in contrast and noise, thus maintaining CNR nearly constant.

We acknowledge the following limitations of the study. First, a relatively small number of patients were included in each group. Second, the diagnostic performance of low-dose cervical spine CT with IR was not assessed. However, CT is a recognised imaging modality in the diagnosis of cervical disc herniation and/or spondylosis [1-3]. Moreover, the overall diagnostic image quality was found to be better with IR (Fig. 5e). Further studies with surgical correlation are necessary to assess the diagnostic accuracy of this new technique. Third, we only evaluated the strength level of IR recommended by the manufacturer (i.e. SAFIRE 3). Further studies are needed to determine the optimal strength level of IR.

In conclusion, the application of IR in cervical spine CT decreases image noise, increases SNR and CNR, and enhances the conspicuity of some anatomical structures. Lowdose CT of the cervical spine with IR provides better image quality of the intervertebral discs, the content of neural foramina, and ligaments compared with standard-dose CT with FBP, while reducing radiation dose by approximately $40 \%$. However, the former protocol provides lower image quality of the soft tissues and vertebrae. Further studies are necessary to determine the optimal strength level of IR, which may depend on the anatomical structure to be analysed.
Acknowledgements The authors thank Julien G. Ott for his help with the noise power spectrum analysis.

Conflict of interest The authors declare that they have no conflict of interest.

Funding or grant None

\section{References}

1. Freund M, Sartor K. Degenerative spine disorders in the context of clinical findings. Eur J Radiol. 2006;58:15-26.

2. Douglas-Akinwande AC, Rydberg J, Shah MV, et al. Accuracy of contrast-enhanced MDCT and MRI for identifying the severity and cause of neural foraminal stenosis in cervical radiculopathy: a prospective study. AJR Am J Roentgenol. 2010;194:55-61.

3. Tins B. Technical aspects of CT imaging of the spine. Insights Imaging. 2010;1:349-59.

4. Biswas D, Bible JE, Bohan M, Simpson AK, Whang PG, Grauer JN. Radiation exposure from musculoskeletal computerized tomographic scans. J Bone Joint Surg Am. 2009;91:18829.

5. Lee TY, Chhem RK. Impact of new technologies on dose reduction in CT. Eur J Radiol. 2010;76:28-35.

6. Tamm EP, Rong XJ, Cody DD, Ernst RD, Fitzgerald NE, Kundra V. Quality initiatives: CT radiation dose reduction: how to implement change without sacrificing diagnostic quality. Radiographics. 2011;31:1823-32.

7. Hoang JK, Yoshizumi TT, Nguyen G, et al. Variation in tube voltage for adult neck MDCT: effect on radiation dose and image quality. AJR Am J Roentgenol. 2012;198:621-7.

8. Leswick DA, Hunt MM, Webster ST, Fladeland DA. Thyroid shields versus z-axis automatic tube current modulation for dose reduction at neck CT. Radiology. 2008;249:572-80.

9. Gervaise A, Louis M, Batch T, et al. Dose reduction at CT of the lumbar spine using a 320-detector row scanner: initial results. J Radiol. 2010;91:779-85.

10. Singh S, Kalra MK, Hsieh J, et al. Abdominal CT: comparison of adaptive statistical iterative and filtered back projection reconstruction techniques. Radiology. 2010;257:373-83.

11. Pontana F, Pagniez J, Flohr T, et al. Chest computed tomography using iterative reconstruction vs filtered back projection (Part 1): evaluation of image noise reduction in 32 patients. Eur Radiol. 2011;21:627-35.

12. Moscariello A, Takx RA, Schoepf UJ, et al. Coronary CT angiography: image quality, diagnostic accuracy, and potential for radiation dose reduction using a novel iterative image reconstruction technique-comparison with traditional filtered back projection. Eur Radiol. 2011;21:2130-8.

13. Winklehner A, Karlo C, Puippe G, et al. Raw data-based iterative reconstruction in body CTA: evaluation of radiation dose saving potential. Eur Radiol. 2011;21:2521-6.

14. Wirth S, Meindl T, Treitl M, Pfeifer KJ, Reiser M. Comparison of different patient positioning strategies to minimize shoulder girdle artifacts in head and neck CT. Eur Radiol. 2006;16:1757-62.

15. Mulkens TH, Marchal P, Daineffe S, et al. Comparison of low-dose with standard-dose multidetector CT in cervical spine trauma. AJNR Am J Neuroradiol. 2007;28:1444-50.

16. Deak PD, Smal Y, Kalender WA. Multisection CT protocols: sexand age-specific conversion factors used to determine effective dose from dose-length product. Radiology. 2010;257:158-66.

17. Bongartz G, Golding SJ, Jurik AG, et al. European guidelines on quality criteria for computed tomography. EUR $16262 \mathrm{http} / / /$ 
www.drs.dk/guidelines/ct/quality/index.htm accessed October 13, 2011.

18. Jones B, Jarvis P, Lewis JA, Ebbutt AF. Trials to assess equivalence: the importance of rigorous methods. BMJ. 1996;313:36-9.

19. Brenner DJ, Hall EJ. Computed tomography-an increasing source of radiation exposure. N Engl J Med. 2007;357:2277-84.
20. Gervaise A, Osemont B, Lecocq S, et al. CT image quality improvement using Adaptative Iterative Dose Reduction with wide-volume acquisition on 320-detector CT. Eur Radiol. 2012;22:295-301.

21. Dougeni E, Faulkner K, Panayiotakis G. A review of patient dose and optimisation methods in adult and paediatric CT scanning. Eur J Radiol. 2012;81:e665-83. 